\title{
Heterogeneity within Autism Spectrum Disorder in Forensic Mental Health: The Introduction of Typologies
}

\begin{tabular}{|r|l|}
\hline Journal: & Advances in Autism \\
\hline Manuscript ID & AIA-08-2016-0021 \\
\hline Manuscript Type: & Research Paper \\
\hline Keywords: & $\begin{array}{l}\text { Autism Spectrum condition, Autism Spectrum Disorder, Behavioural } \\
\text { Phenotypes, Forensic, Intellectual Disability, Mental Disorder }\end{array}$ \\
\hline \multicolumn{2}{|l}{} \\
\hline
\end{tabular}

SCHOLARONE ${ }^{\prime \prime}$

Manuscripts 


\title{
Heterogeneity within Autism Spectrum Disorder in Forensic Mental Health: The Introduction of Typologies
}

\begin{abstract}
Purpose: Individuals with diagnoses of Autism Spectrum Disorder (ASD) within criminal justice settings are a highly heterogeneous group. Although studies have examined differences between those with and without ASD in such settings, there has been no examination of differences within the ASD group.

Design/methodology/approach: Drawing on the findings of a service evaluation project, this paper introduces a typology of ASD within forensic mental health and learning disability settings.

Findings: The eight sub-types that are described draw on clinical variables including psychopathy, psychosis and intensity/ frequency of problem behaviours that co-occur with the ASD. The initial assessment of inter rater reliability on the current version of the typology revealed excellent agreement, multirater $K_{\text {free }}=.90$.

Practical implications: The proposed typology could improve understanding of the relationship between ASD and forensic risk, identify the most appropriate interventions and provide prognostic information about length of stay. Further research to refine and validate the typology is ongoing.
\end{abstract}

Originality/value: This paper introduces a novel, typology based approach which aims to better serve people with ASD within criminal justice settings. 


\section{Introduction}

Prevalence data about offending behaviours amongst people with an autistic spectrum disorder (ASD) suggest that this group is no more at risk than the general population (Mouridsen, Rich, Isager, \& Nedergaard, 2008). However, there is some evidence that people with an ASD may be over represented in specialist forensic mental health or learning disability settings, with reported rates between 1.5 and $30 \%$ (Scragg \& Shah, 1994; Alexander et al., 2011; Esan, Chester, Gunaratna, Hoare, \& Alexander, 2015). Prevalence estimates are unavailable for prison, as there is currently no policy of routine screening, yet people with ASD also appear over represented (Underwood, Forrester, Chaplin, \& McCarthy, 2013). It has been suggested that people with ASD experience disadvantage when interacting with criminal justice agencies, particularly during police interviews (North, Russell, \& Gudjonsson, 2008; Archer et al., 2013) and in court (Allely, 2015). Those with adequate language skills may not initially appear vulnerable, meaning that police and courts fail to provide support to assist with communication and protect the individual's rights. Archer et al. (2013) note that often defendants with ASD present with a lack of empathy or remorse and hence may be sentenced more harshly.

Woodbury-Smith et al. (2005) highlighted that when considering vulnerability to offending by people with ASD, specific ASD factors, such as poor social understanding or circumscribed interests, difficulties in adjusting to the diagnosis, and the impact of social exclusion are relevant. The sensory issues often present in ASD can also directly impact risk of violence on occasion (e.g. Mawson, Grounds, \& Tantam, 1985). However, WoodburySmith et al., 2005) noted that risk factors identified in general criminological literature are also relevant, such as low IQ, poor school achievement, truancy, aggressive behaviour, and hyperactivity-impulsivity-inattention. This sentiment is echoed by Berney and Pierce (2016) who stated that automatically assuming a causal relationship between ASD and an individuals' offending behaviour is a reductionist approach which can overshadow other pertinent risk factors for offending, and subsequently, a lack of tailored treatment. Mouridsen et al. (2008) suggested that neurocognitive problems and associated psychiatric illness are important risk factors to consider. Indeed, antisocial personality disorder has been noted amongst offenders with ASD (Dein \& Woodbury-Smith, 2010; Långström, Grann, Ruchkin, Sjöstedt, \& Fazel, 2009). It is possible that such difficulties remain undiagnosed if a diagnosis of ASD is present. Långström et al. (2009) reported that people with ASD who had committed violent crimes were more likely to have comorbid psychosis, substance misuse and personality disorder.

Questions remain regarding the role of psychopathy, which increases the probability of socially deviant behaviour in affected individuals, and can co-occur in ASD (Jones et al., 2009). Woodbury-Smith and colleagues (2005) reported that offenders with ASD had an impaired ability to recognise fear compared with non-offenders with ASD, which could suggest co-morbid psychopathy. Despite this, some behaviours that are seen in both individuals with psychopathy and those with ASD, may appear superficially similar (e.g. unemotionality and behavioural dyscontrol); however, the neurocognitive underpinnings may be quite different (Rogers, Viding, Blair, Frith, \& Happé, 2006). Indeed, Rogers et al. (2006) suggest that different aspects of empathy are impaired in individuals with ASD traits and those with psychopathic traits(Department of Health, 2012). Whilst individuals with ASD have difficulty in understanding what others think ('cognitive empathy'), individuals with psychopathic traits have difficulty in resonating with other people's feelings ('affective empathy'). Resonating with other people's feelings is thought to be particularly important for 
feeling true empathy towards others' suffering. This suggests that psychopathy is a particularly important feature to consider when assessing and treating forensic mental health problems, while autistic symptomatology, although related, may be of less clinical importance in some individuals.

The idea that specific neurocognitive problems and comorbid psychopathology can explain forensic risk in this population is somewhat reflected in the studies which examined the specific clinical and forensic profiles of those with ASD within forensic services, as compared to those without. For example, Murphy (2003) reported no high-secure hospital patients with Asperger syndrome had any history of serious antisocial behaviour or criminal convictions before age 18. It has been noted that offenders with ASD perpetrated significantly more violent or sexual crimes against people, but less property offences (Cheely et al., 2012; Kumagami and Matsuura, 2009). Haw, Radley and Cooke (2013) compared 51 male forensic patients with ASD in a low-secure forensic psychiatry service, to 43 patients without ASD. The ASD group were younger (27 vs. 33 years) and younger at their first contact with psychiatric services. Those with ASD were more likely to be admitted from prison or courts, rather than civil pathways, suggesting more serious level of offending. Over $75 \%$ had a history of physical violence, and a third had convictions for serious violence or homicide. Offending behaviour was described as atypical, involving uncommon offences, e.g. harassment or stalking. Esan et al. (2015) conducted a similar study in a forensic intellectual disability population, and found that patients with ASD had similar rates of convictions in broad categories of violence, arson and sexual offending. In terms of clinical comorbidity, Haw reported that almost $75 \%$ of those with ASD had psychiatric comorbidity, most commonly schizophrenia, and $4.4 \%$ had personality disorders. Drug and alcohol disorders were uncommon, although many had histories of misuse. Similarly, Esan et al. (2015) found that although comorbidity rates were lower than the comparison group, those with ASD had a wide range of comorbid diagnoses, including psychosis (14\%), bipolar disorder $(10 \%)$, substance misuse (12\%), personality disorder $(36 \%)$, and epilepsy $(26 \%)$. Woodbury-Smith et al., 2005) reported that $19 \%$ of an offender group with Asperger's syndrome met antisocial personality disorder criteria.

Furthermore, this idea has considerable implications for the inpatient care-pathway of patients with ASD detained in hospital. For example, patients with co-morbid psychopathy will require intensive longer term support, while those with psychosis illnesses may require shorter stays, and different clinical interventions. At present, we know very little about the relationship between these constructs and forensic risk. While NICE (2012) define generic care-pathways for autism, incorporating psychosocial and biomedical interventions, there are no specific care-pathways described for people with ASD who are detained within hospital because of forensic mental health problems. Furthermore, while there are a limited number of specialist ASD forensic units for individuals with an ASD, individuals are most often placed among 'neurotypical' offenders and expected fit in with conventional therapeutic programmes (Murphy, 2010), which are primarily developed for patients with personality and psychotic disorders.

Unsurprisingly then, patients and prisoners with an ASD diagnosis have been described as presenting clinical challenges, and difficult to engage therapeutically (Murphy, 2010). These include empathy deficits, cognitive rigidity and problems with central coherence (a tendency to become fixed with specific details rather than an awareness/appreciation of the wider context), a lack of appreciation of the emotional and social consequences of their actions, egocentric view of the world, and dysfunctional coping strategies adopted by individuals to 
deal with interpersonal conflict. Esan et al. (2015) reported that patients with ASD had significantly higher rates of self-harm, physical intervention, seclusion, enhanced observations and as required medication, which could indicate higher levels of behavioural and mental health difficulties. In prison, people with ASD experience a range of difficulties (Underwood et al., 2013), including impairment of social communication and issues of sensory over- or under-stimulation (Robinson et al., 2012). Interpersonal difficulties are frequent, with people with ASD being perceived as inappropriate, discourteous or even confrontational to staff and other prisoners (McAdam, 2012).

Sensory issues are prevalent, with the prison environment experienced as noisy, brightly lit and enclosed (Underwood et al., 2013). It has also been suggested that prisoners with ASD include being locked in their cell for longer than other prisoners for their own safety (Myers, 2004), due to vulnerability to bullying or exploitation (Cashin \& Newman, 2009; McAdam, 2012). Conversely, it has been noted that traditional markers of therapeutic progress used within forensic services, such as stable behaviour, may not evidence the same progress in those with ASD (Dein \& Woodbury-Smith, 2010), due to exemplary behaviour in a particular environment (Lorna Wing, 1997). There have been case reports outlining how people with ASD who have forensic mental health problems present with marked complexity and as a consequence may be excluded from services (Baliousis, Vollm, Banerjee, \& Duggan, 2013). Forensic risk assessment and management often does not consider factors associated with autism (Gunasekaran, 2013). It is therefore likely that inpatients and prisoners with ASD may experience poorer outcomes following admission or imprisonment within forensic settings. However, research is lacking, and no studies have examined outcomes of those with ASD from generic forensic psychiatry services or prison.

\section{A Case for a Typology?}

In recent years, there has been an increasing move towards the accurate prediction of treatment outcomes, in line with the payment by results $(\mathrm{PbR})$ agenda (Bhaumik, Devapriam, Gangadharan, Hiremath, \& Roy, 2011). A recent service evaluation project of a specialist forensic service for people with an intellectual disabilities showed that a clinical diagnosis of ASD was not significantly associated with good or adverse treatment outcomes measured by length of stay, or direction of care pathway (Alexander et al., 2011; Esan et al., 2015). This suggests that treatment outcomes from secure hospital settings are not necessarily mediated or moderated by the single categorical ASD diagnosis, but rather by a combination of different variables in which the categorical ASD diagnosis is only one. This is in keeping with the earlier views about the wide heterogeneity of the ASD group (Murphy, 2007).

It was therefore felt that there was a need for a clinically useful typology of patients who had a diagnosis of ASD within forensic hospital settings. The aims of such a typology would include:

1. Improving our understanding of the relationship between ASD and forensic risk.

2. Helping to identify which constructs relate to our understanding of forensic risk, which can be used to help inform care pathways.

3. Improving our understanding of the aetiology and prognosis of forensic mental health problems amongst people with ASD.

4. Improving the hospital care-pathway for patients with ASD, which may lead to shorter hospital stays for them and cost savings for those who commission services. 
The typology first arose from the authors' clinical experience within forensic intellectual disability services. Based on a series of multi-disciplinary discussions involving eight professionals (four psychiatrists, two psychologists, two nurses) within the team where the service evaluation was conducted (Esan et al., 2015), four types of patients were described. These types are elaborated on below with the help of illustrative examples. The examples are not of real patients, but are representative of the sort of the clinical presentations that one sees in this area.

\section{The misdiagnosed, medium intensity and medium frequency group (The M-MIMF group):}

$\mathrm{Mr} \mathrm{AB}$ is a 27 year old man who was admitted to hospital when he was facing charges of criminal damage and assault. This was the culmination of a similar pattern of behaviour for several years. He was variously described as having a personality disorder, mild learning disability, psychosis and was often a subject of boundary disputes between services because of these different diagnoses. He was described as stubborn and wilful, intolerant of others, having poor frustration tolerance, demanding that people do things for him then and there, resistant to change, intolerant of noise while himself being quite noisy and often responding with a disproportionate degree of arousal for small changes that others would consider trivial. His behavioural difficulties were of moderate to severe intensity and would happen at a frequency of around once or twice a week. During these episodes it was very difficult to deescalate or calm him down. Within the hospital setting, he underwent a systematic assessment and was found to have an ASD. He had no other diagnosis. The intervention focused on the SPELL approach (Smeardon, 1998), maintaining structure and consistency and psycho-education of the staff group around him. His behaviour improved and it was possible to move him on to conditions of lower security within six to eight months.

\section{The unemotional, high intensity but low frequency group (The U-HILF group):}

$\mathrm{CD}$ is a 47 year old man who functions in the borderline intellectual range. From a young age, he was found to be odd, had few friends and in his teenage years received the diagnosis of ASD. He had a fascination with hunting knives and could talk in a very matter of fact way about the ways in which they are made, the companies that made them, all the places where double murders had happened using particular types of these knives, etc. At the age of 25, he was convicted for the murder of an acquaintance who he had invited into his flat. He said he wanted to see what facial expression people would have when they were bleeding from a neck wound and hence he had spiked his acquaintance's drink with Diazepam and then carried out the offence. He describes all this in a very unemotional and detached manner. Within the hospital setting, he underwent a systematic assessment and was found to have an ASD and PCL-SV scores (Hart, Cox, \& Hare, 1995) just above the cut off. After years of therapy, he now says that he understands it "cannot have been pleasant" for his victim. CD is a model patient on the ward and has not been involved in any violence. Psychology reports continue to emphasise the importance of robust external supervision and while it has been possible to discharge him to conditions of lower security, he remains an in-patient after 22 years.

\section{The difficult, high intensity and high frequency group (The D-HIHF group):}

$\mathrm{Mr}$ EF is a 23 year old man who has a mild learning disability. In early childhood, he was diagnosed as having ASD and a hyperkinetic conduct disorder. There were reports of cruelty to animals, unprovoked violence against classmates and family members, destruction of property, fire setting, self-harming and other anti- social behaviours. As he became a young adult these problems became unmanageable at home. The local authority found him a 
community placement that broke down within a few weeks after he assaulted staff and caused extensive property damage. Since then, he has been in various hospitals of low to medium security. In addition to his learning disability, he is also diagnosed with adult ADHD and emotionally unstable personality disorder. His behaviour on the ward poses problems on a daily basis and large numbers of staff members have been injured by him. His last three placements gave him notice and it has been almost impossible to find new placements.

\section{The psychosis-variable intensity variable frequency group (The P-VIVF group):}

$\mathrm{Mr}$ GH and Mr IJ have very similar histories. They were both diagnosed as having mild learning disability and ASD at a young age. Both of them started having behavioural problems; aggression towards people and property and threatening behaviours in their early adulthood. For quite some time, it was assumed that these behaviours were related to their ASD and learning disability. Following convictions for seriously assaulting strangers who they thought were being "nasty" to them, they received hospital orders for treatment in secure hospitals. A detailed assessment within the hospital setting suggested that they had a psychotic illness characterised by paranoid thoughts and hallucinatory experiences. They were treated with antipsychotic medication. $\mathrm{Mr} \mathrm{GH}$ responded well to this, his risk behaviours reduced and while he continued to have the ASD features and related difficulties, it was possible to manage him using the Structure, Positive, Empathy, Low arousal, Links (SPELL) principles and move him on to a lower level of security within 14 months of admission. Mr IJ's psychotic symptoms did not respond that well and he continued to have serious aggressive outbursts of the kind that brought him to hospital. He improved slightly on Clozapine, although after over three years in hospital, he still has not reached a stage where a safe discharge to lower levels of security can be considered.

These provisional types and descriptions were presented by the authors (RA, IG, SH) at three regional and national meetings attended by interdisciplinary peers, and discussed further with five outside experts (two psychiatrists, two psychologists, one professor of forensic psychiatry). From these discussions, it was clear that these types were distinguished by three main variables: the presence or absence of psychosis, the presence or absence of callous, unemotional or psychopathic personality traits, and the frequency and severity of behaviour. The typologies were refined further based on this, and the current version of the typology is presented in Figure 1.

In order to classify an individual patient, the clinican would first assess the level of psychopathic traits using the The Psychopathy Checklist- Screening Version (PCL-SV) (Hart, Cox \& Hare, 1995), and place the individual in a category of either low or high psychopathy. Psychopathy in this context is seen as a proxy for the callous, unemotional traits description in the original examples. Of the low psychopathy group, if psychosis is present, the patient is classified as Type 1: low psychopathy plus psychosis; and if psychosis is not present; as Type 2: low psychopathy and no psychosis. Of the high psychopathy group; if psychosis is present, the patient is classified as Type 3: high psychopathy plus psychosis; and if psychosis is not present as Type 4: high psychopathy and no psychosis. Each of these four groups can have an (a) and (b) sub groups, relative to the frequency of behavioural problems; with a) denoting lower level, and b) denoting higher level behavioural problems. Thus overall, there are eight potential subtypes, as follows:

Type 1: low psychopathy and psychosis

1a low level behaviour

$1 \mathrm{~b}$ high level behaviour 
Type 2: low psychopathy and no psychosis; 2a low level behaviour

$2 \mathrm{~b}$ high level behaviour

Type 3: high psychopathy and psychosis;

3a low level behaviour

$3 \mathrm{~b}$ high level behaviour

Type 4: high psychopathy and no psychosis

4a low level behaviour

$4 \mathrm{~b}$ high level behaviour

There are hypothesised differences between these subtypes in terms of their clinical presentation and neurocognitive functioning, which has associated implications for treatment and care pathways. For example, for those with low psychopathy, psychosis, and low frequency behavioural difficulties, the priority would be the successful treatment of their mental health problems, suggesting a relatively shorter length of hospital stay. For those with low psychopathy, who do not have psychosis, but have higher behavioural difficulties, there is likely to be a need for careful interventions and staff training, based upon the SPELL approach, but again, hospital stay may be shorter because their difficulties are associated with autism, rather than comorbid psychopathy. Those with comorbid psychopathy are likely to require careful supervision, but this may not have to be within medium or high security, and will vary according to whether they have psychosis and the frequency and intensity of their behavioural difficulties. For example, the group with psychopathy and no psychosis, plus lower behaviour difficulties, are likely to engage in offending behaviours linked to their circumscribed interests, which may be unpredictable at times, but they may be relatively easy to manage. They are likely to have some features of psychopathy, but as this is a spectrum, their difficulties may not be as marked. For those who have higher levels of psychopathy, and behaviour problems, they are likely to require a high degree of careful management within conditions of security because of behavioural problems; they are likely to need the longest length of stay within hospital.

The revised typology was felt to have good face validity and was hence incorporated into the routine clinical practice within the teams that participated in the Esan et al. (2015) service evaluation. The three psychiatrists involved in the original service evaluation independently assigned the 23 patients with ASD within the service into these subtypes and showed excellent agreement with a multirater $K_{\text {free }}=.90$.

Figure 1 


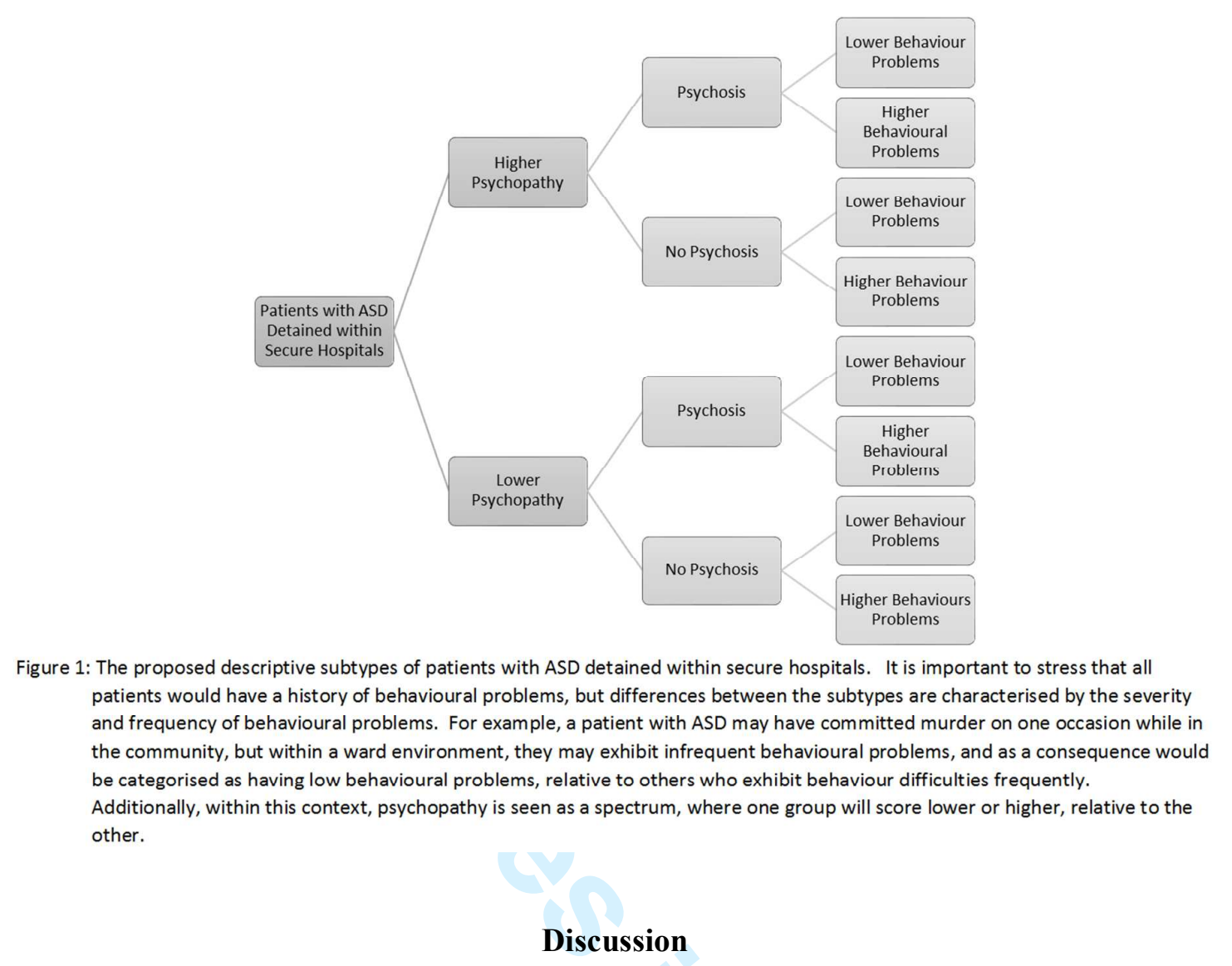

There are currently a number of problems with the evidence base and clinical practice in regards to the small but significant population of people with ASD within criminal justice settings. Typologies are widely used within healthcare and have been previously developed in relation to ASD by Lorna Wing (1996), who proposed four subtypes within ASD based on levels of social engagement and the pattern of social impairment. The typology that has been introduced here is an initial attempt to better capture the well-known heterogeneity of this population. While careful, individualised diagnostic, and psychological formulations are of utmost importance when assessing the clinical needs and forensic risk of this under researched group, it is possible that classifying patients into typologies could have numerous benefits, such as improving our understanding of the relationship between ASD and forensic risk, informing the most appropriate treatment pathways, and the prediction of treatment outcomes.

This paper has introduced such a typology, which was directly developed from the experience of practising clinicians. The typologies appear to have some face validity and inter-rater reliability. However, it is acknowledged the proposed subtypes need to be examined further. A significant body of future research funded by the National Institute for Health Research is currently under way to further evaluate and refine the typology (Langdon, n.d.; https://research.kent.ac.uk/match/about-us-2/). There are three separate work-streams within the planned research; one of which is a consultation exercise, via focus groups with clinicians from inpatient services, people with ASD, and family members and carers. This will seek comments on the validity of the subtypes and whether any further characteristics need to be considered. Following on from this, and incorporating any changes, clear descriptions of each of our subtypes will be developed. A further consultation and consensus rating exercise will 
ask clinicians to assign vignettes to our proposed subtypes independently and ratings compared. Clinicians will be asked to give their expert opinion regarding the subtypes and provide any feedback, which will be used to further refine our subtypes. A final summary of each subtype will be produced which will be used to categorise patients. Data will be collected on a variety of demographic and clinical variables; and the validity of the subtypologies will be examined by comparing data on patient and hospital variables between patients characterised according to the subtypes, using a cross-sectional design. This group of patients will be followed up, and behavioural data collected over time.

It is hoped that this future research will further develop understanding of the relationship between ASD and forensic risk, improve our understanding of the aetiology and prognosis of forensic mental health problems amongst people with ASD, better direct the most appropriate clinical interventions, and support the commissioning process.

\section{References}

Alexander, R., Hiremath, A., Chester, V., Green, F., Gunaratna, I., \& Hoare, S. (2011). Evaluation of treatment outcomes from a medium secure unit for people with intellectual disability. Advances in Mental Health and Intellectual Disabilities, 5(1), 22-32. http://doi.org/10.5042/amhid.2011.0013

Allely, C. (2015). Autism spectrum disorders in the criminal justice system : police interviewing, the courtroom and the prison environment. Recent Advances in Autism.

Archer, N., Hurley, E. A., Raggi, C., Xenitidis, K., Moisan, M., Deeley, Q., ... Is, H. (2013). A justice system failing the autistic community. Journal of Intellectual Disabilities and Offending Behaviour, 4(12), 42-52. http://doi.org/10.1108/JIDOB-02-2013-0003

Baliousis, M., Vollm, B. A., Banerjee, P., \& Duggan, C. (2013). Autistic spectrum disorder, personality disorder and reading disability: a complex case that falls between the cracks? The Journal of Forensic Psychiatry \& Psychology, 24(2), 286-292. http://doi.org/10.1080/14789949.2013.766231

Cashin, A., \& Newman, C. (2009). Autism in the criminal justice detention system: A review of the literature. Journal of Forensic Nursing, 5(2), 70-75. http://doi.org/10.1111/j.1939-3938.2009.01037.x

Cheely, C. A., Carpenter, L. A., Letourneau, E. J., Nicholas, J. S., Charles, J., \& King, L. B. (2012). The prevalence of youth with Autism spectrum disorders in the criminal justice system. Journal of Autism and Developmental Disorders, 42(9), 1856-1862. http://doi.org/10.1007/s10803-011-1427-2

Dein, K., \& Woodbury-Smith, M. (2010). Asperger syndrome and criminal behaviour. Advances in Psychiatric Treatment, 16(1), 37-43. http://doi.org/10.1192/apt.bp.107.005082

Department of Health. (2012). Transforming care: A national response to Winterbourne View Hospital.

Esan, F., Chester, V., Gunaratna, I. J., Hoare, S., \& Alexander, R. T. (2015). The clinical, forensic and treatment outcome factors of patients with autism spectrum disorder treated in a forensic intellectual disability service. Journal of Applied Research in Intellectual Disabilities : JARID, 28(3), 193-200. http://doi.org/10.1111/jar.12121

Gunasekaran, S. (2013). Assessment and management of risk in autism. Advances in Mental Health and Intellectual Disabilities, 6(6), 314-320. http://doi.org/10.1108/20441281211285964

Hart, S. D., Cox, D. N., \& Hare, R. D. (1995). The Psychopathy Checklist- Screening 
Version (PCL-SV). Toronto, Ontario, Canada: Multi-Health Systems.

Haw, C., Radley, J., \& Cooke, L. (2013). Characteristics of male autistic spectrum patients in low security: are they different from non-autistic low secure patients? Journal of Intellectual Disabilities and Offending Behaviour, 4(1/2), 24-32. http://doi.org/10.1108/JIDOB-03-2013-0006

Jones, A. P., Larsson, H., Ronald, A., Rijsdijk, F., Busfield, P., Mcmillan, A., ... Viding, E. (2009). Phenotypic and Aetiological Associations Between Psychopathic Tendencies, Autistic Traits, and Emotion Attribution. Criminal Justice and Behavior, 36(11), 11981212. http://doi.org/10.1177/0093854809342949

Kumagami, T., \& Matsuura, N. (2009). Prevalence of pervasive developmental disorder in juvenile court cases in Japan. Journal of Forensic Psychiatry \& Psychology, 20(906461227), 974-987. http://doi.org/10.1080/14789940903174170

Langdon, P. (n.d.). People with AuTism detained within hospitals: defining the population, understanding aetiology and improving Care patHways (The mATCH study). Available from: http://www.hra.nhs.uk/news/research-summaries/the-match-study/. Accessed $1^{\text {st }}$ August 2016.

Långström, N., Grann, M., Ruchkin, V., Sjöstedt, G., \& Fazel, S. (2009). Risk factors for violent offending in autism spectrum disorder: a national study of hospitalized individuals. Journal of Interpersonal Violence, 24(8), 1358-70. http://doi.org/10.1177/0886260508322195

Mawson, D. C., Grounds, A., \& Tantam, D. (1985). Violence and Asperger's syndrome: A case study. The British Journal of Psychiatry, 147(5), 566-569. http://doi.org/10.1192/bjp.147.5.566

McAdam, P. (2012). Knowledge and understanding of the autism spectrum amongst prison staff. Good Autism Practice, 10(1), 19-25.

Mouridsen, S. E., Rich, B., Isager, T., \& Nedergaard, N. J. (2008). Pervasive developmental disorders and criminal behaviour: a case control study. International Journal of Offender Therapy and Comparative Criminology, 52(2), 196-205. http://doi.org/10.1177/0306624X07302056

Murphy, D. (2003). Admission and cognitive details of male patients diagnosed with Asperger's Syndrome detained in a Special Hospital: comparison with a schizophrenia and personality disorder sample. Journal of Forensic Psychiatry \& Psychology, 14(3), 506-524. http://doi.org/10.1080/1478994031000152736

Murphy, D. (2007). Hare Psychopathy Checklist Revised profiles of male patients with Asperger's syndrome detained in high security psychiatric care. Journal of Forensic Psychiatry \& Psychology, 18(1), 120-126. http://doi.org/10.1080/14789940601014777

Murphy, D. (2010). Understanding offenders with autism-spectrum disorders: what can forensic services do?: Commentary on... Asperger Syndrome and Criminal Behaviour. Advances in Psychiatric Treatment, 16(1), 44-46. http://doi.org/10.1192/apt.bp.109.006775

Myers, F. (2004). On the Borderline? People with Learning Disabilities and/or Autistic Spectrum Disorders in Secure, Forensic and Other Specialist Settings. Edinburgh.

NICE. (2012). Autism Spectrum Disorder in adults: diagnosis and management.

North, A. S., Russell, A. J., \& Gudjonsson, G. H. (2008). High functioning autism spectrum disorders: an investigation of psychological vulnerabilities during interrogative interview. Journal of Forensic Psychiatry \& Psychology, 19(3), 323-334. http://doi.org/10.1080/14789940701871621

Robinson, L., Spencer, M. D., Thomson, L. D. G., Stanfield, A. C., Owens, D. G. C., Hall, J., \& Johnstone, E. C. (2012). Evaluation of a screening instrument for autism spectrum disorders in prisoners. PLoS ONE, 7(5), e36078. 
http://doi.org/10.1371/journal.pone.0036078

Rogers, J., Viding, E., Blair, R. J., Frith, U., \& Happé, F. (2006). Autism spectrum disorder and psychopathy: shared cognitive underpinnings or double hit? Psychological Medicine, 36(12), 1789-1798. http://doi.org/10.1017/S0033291706008853

Sabyasachi Bhaumik; John Devapriam; Satheesh Gangadharan; Avinash Hiremath; Ashok Roy. (2011). Payment by results for learning disability services: a model for the future? Advances in Psychiatric Treatment, 17(6), 470-475.

Scragg, P., \& Shah, A. (1994). Prevalence of Asperger's syndrome in a secure hospital. British Journal of Psychiatry, 165, 679-682. http://doi.org/10.1192/bjp.165.5.679

Underwood, L., Forrester, A., Chaplin, E., \& McCarthy, J. (2013). Prisoners with neurodevelopmental disorders. Journal of Intellectual Disabilities and Offending Behaviour, 4(1), 17-23. http://doi.org/10.1108/JIDOB-05-2013-0011

Wing, L. (1996). The Autistic Spectrum: A Guide for Parents and Professionals. London: Constable.

Wing, L. (1997). Asperger's syndrome: Management requires diagnosis. Journal of Forensic Psychiatry, 8(2), 253-257. http://doi.org/10.1080/09585189708412008

Woodbury-Smith, M. R., Clare, I. C. H., Holland, A. J., Kearns, A., Staufenberg, E., \& Watson, P. (2005). A case-control study of offenders with high functioning autistic spectrum disorders. Journal of Forensic Psychiatry \& Psychology, 16(4), 747-763. http://doi.org/10.1080/14789940500302554 